\title{
Meshless solution of the neutron diffusion equation by the RBF collocation method using optimum
}

\section{shape parameters}

\section{Tayfun Tanbay iD}

Bursa Technical University, Department of Mechanical Engineering, 16330 Bursa, TURKEY

\section{Cite this paper as:}

Tanbay,T.,(2019). Meshless solution of the neutron diffusion equation by the RBF collocation method using optimum shape parameters. Journal of Innovative Science and Engineering 3(1): 23-31.

*Corresponding author: Tayfun Tanbay E-mail: tayfun.tanbay@btu.edu.tr Tel: +90 (224) 3003770

Received Date: 27/05/2019

Accepted Date: 17/06/2019

(C) Copyright 2018 by

Bursa Technical University. Available online at http://jise.btu.edu.tr/

\section{(c) (i) (8)}

The works published in Journal of Innovative Science and Engineering (JISE) are licensed under a Creative Commons Attribution-NonCommercial 4.0 International License.

\begin{abstract}
The meshless radial basis function collocation method is an efficient numerical technique for solving partial differential equations. The multiquadric is the most widely utilized radial function for this purpose; but it contains a shape parameter, which has a significant effect on the performance of the method. In this study, the meshless collocation method employing multiquadric as the radial function with optimum shape parameters is applied to the numerical solution of the multigroup neutron diffusion equation. The optimization of the shape parameter is performed by minimizing the Madych-Nelson function. One external and two fission source problems are solved to investigate the performance of the method. The results show that the meshless collocation method with optimized shape parameters yield a high level of accuracy with an exponential convergence rate.

Keywords: Meshless, RBF, Collocation, Optimum shape parameter, Neutron diffusion.
\end{abstract}




\section{Introduction}

Meshless methods have become a powerful alternative class of numerical methods to conventional techniques such as finite element, boundary elements and finite difference methods in solving partial, integral and integro-differential equations. The radial basis function (RBF) collocation method is a prominent member of meshless methods owing to its truly meshless nature, exponential convergence rate and ease of implementation. The method was first proposed by Kansa to solve hydrodynamics problems [1] and since then, it has been applied to many fields of engineering including heat transfer, structural mechanics, optics, and so on.

Although there exists several radial functions in literature, the generalized multiquadric and Gaussian functions are the most widely utilized RBFs within the collocation method. These functions contain a term, called the shape parameter, which has an important role on the performance of the RBF collocation method. The shape parameter affects significantly the accuracy, stability and convergence rate of the meshless RBF collocation technique. In the case of function interpolation with the multiquadric, it was proved theoretically that the approximation error vanishes as the shape parameter goes to infinity if an infinite precision computation can be performed [2]. However, in practice as the shape parameter increases the error decreases first, but then it tends to increase when a certain value of this parameter is exceeded due to the ill conditioning of the interpolation matrix. This type of trade-off between accuracy and stability is also observed in RBF collocation solution of differential equations and it suggests that an optimum value of the shape parameter exists, which yields highly accurate solutions without causing instability in the numerical solution.

Optimization of the shape parameter has drawn the attention of many researchers since the introduction of RBF collocation method for solving differential equations. Early studies focused on the number of nodes, and several formulae relating the optimum value of the shape parameter to this factor were proposed [3-5]. Rippa observed that the optimum value of the shape parameter depends on the collocation matrix and interpolation function, and proposed an optimization method based on the minimization of a function, which behaves similarly to the root mean square error of the numerical solution [6]. Also, there exist approaches utilizing genetic algorithm [7] and particle swarm optimization [8] for calculating the optimum shape parameter. The optimization problem was solved recently, and Luh determined the criteria to calculate the optimum value of the shape parameter for the generalized multiquadric function [9]. The optimization method is based on minimizing a function, namely the Madych-Nelson function, and it is shown to be effective for interpolation of functions.

The neutron diffusion equation governs the behavior of neutrons in a multiplying or non-multiplying nuclear system. This equation was solved by the RBF collocation method [10-12]. Although an exponential convergence rate and better accuracy than linear finite and boundary element solutions were found [11], the use of constant shape parameters led to an ill-conditioning and accuracy degradation when a certain shape parameter value was exceeded. The present study deals with the numerical solution of the two-dimensional multi-group neutron diffusion equation in homogeneous media with the RBF collocation method utilizing optimum shape parameters. The multiquadric function is chosen as the radial function, and the potential of the meshless collocation method in producing high fidelity numerical solutions is demonstrated. 


\section{Numerical Formulation}

The two-dimensional multi-group neutron diffusion equation and its boundary conditions take the following form for a square domain with reflective type boundary on bottom and left sides, vacuum type boundary on top and right sides:

$$
\begin{aligned}
& \sum_{g^{\prime}=1}^{g-1} \Sigma_{s, g^{\prime} \rightarrow g} \phi_{g^{\prime}}^{(n)}+s_{g}^{(n-1)}, \quad 0 \leq x, y \leq a \\
& \frac{\partial \phi_{g}}{\partial y}(x, 0)=0, \quad 0 \leq x<a \\
& \phi_{g}(a, y)=0, \quad 0 \leq y<a \\
& \phi_{g}(x, a)=0, \quad 0<x \leq a \\
& \frac{\partial \phi_{g}}{\partial x}(0, y)=0, \quad 0<y \leq a
\end{aligned}
$$

Here $g=1, \ldots, G$ is the energy group, $\phi_{g}$ is the group neutron flux, $\Sigma_{r, g}$ is the group removal cross section, $\Sigma_{s, g^{\prime} \rightarrow g}$ is the group-to-group scattering cross section, $D_{g}$ is the group diffusion constant, $a$ is the size of the domain, and $n$ is the iteration index. In Eq. (1), it is assumed that there is no upscattering of neutrons. The source term, $s_{g}^{(n-1)}$, depends on the physical configuration of the problem:

$$
s_{g}^{(n-1)}=\left\{\begin{aligned}
\frac{\chi_{g}}{k^{(n-1)}} \sum_{g^{\prime}=1}^{G} v_{g^{\prime}} \Sigma_{f, g^{\prime}} \phi_{g^{\prime}}^{(n-1)}, & \text { Multiplying medium } \\
s_{g, \text { ext }}, & \text { Nonmultiplying medium }
\end{aligned}\right.
$$

In Eq. (3), $\chi_{g}$ is the group fission spectrum function, $k$ is the multiplication factor, $v_{g^{\prime}}$ is the number of fission neutrons emitted in group $g^{\prime}, \Sigma_{f, g^{\prime}}$ is the group fission cross section, and $s_{g, e x t}$ is an external source term. For a multiplying medium (i.e., fission source) the neutron diffusion equation is solved iteratively by the fission source iteration [11], whereas a direct solution can be obtained if a nonmultiplying medium exists.

The formulation of the numerical method starts by introducing a set of interpolation nodes

$$
\begin{aligned}
& D=\left\{\boldsymbol{x}_{1}, \ldots, \boldsymbol{x}_{N_{D}}\right\} \\
& B_{V}=\left\{\boldsymbol{x}_{N_{D}+1}, \ldots, \boldsymbol{x}_{N_{D}+N_{V}}\right\} \\
& B_{R}=\left\{\boldsymbol{x}_{N_{D}+N_{V}+1}, \ldots, \boldsymbol{x}_{N_{D}+N_{V}+N_{R}}\right\} \\
& E=\left\{\boldsymbol{x}_{N_{D}+N_{B}+1}, \ldots, \boldsymbol{x}_{N_{D}+N_{B}+N_{E}}\right\}
\end{aligned}
$$


where $\boldsymbol{x} \equiv(x, y), N_{D}, N_{V}, N_{R}$ and $N_{E}$ represent the number of domain, vacuum boundary, reflective boundary and external nodes, respectively while $N_{B}=N_{V}+N_{R}$ is the number of total boundary nodes. The purpose of using external nodes is to improve the accuracy of the collocation method by satisfying the governing differential equation on both the domain and the boundary [13].

The next step of the numerical solution is expanding the group neutron flux into a finite series of radial basis functions

$$
\phi_{g}(x, y) \cong \sum_{j=1}^{N_{T}} a_{j, g} \psi_{j}(x, y)
$$

where $a_{j, g}, j=1, \ldots, N_{T}, g=1, \ldots, G$ are the coefficients to be determined, $\psi_{j}, j=1, \ldots, N_{T}$ are the RBFs, and $N_{T}=$ $N_{D}+N_{B}+N_{E}$ is the total number of interpolation nodes.

The generalized multiquadric radial basis function is expressed as

$$
\psi_{j}(x, y)=\left[\left(x-x_{j}\right)^{2}+\left(y-y_{j}\right)^{2}+c^{2}\right]^{q}
$$

where $c$ is the shape parameter and $q$ is the exponent of the generalized multiquadric function. In this study, the multiquadric is chosen as the RBF (i.e., $q=1 / 2$ ), and the value of the shape parameter is determined by minimizing the Madych-Nelson function given by [9]

$$
M N(c)= \begin{cases}\sqrt{\frac{8 \rho \xi^{(1+\theta+d) / 2} \exp (c \xi)}{\exp \left(\xi^{2} / \sigma\right)}} c^{(\theta-d-1) / 4}\left(\frac{2}{3}\right)^{c /(24 \rho h)}, & c \in[24 \rho h, 12 a \rho) \\ \sqrt{\frac{2 \xi^{(1+\theta+d) / 2} \exp (c \xi)}{3 a \exp \left(\xi^{2} / \sigma\right)}} c^{(1+\theta-d) / 4}\left(\frac{2}{3}\right)^{a /(2 h)}, & c \in[12 a \rho, \infty)\end{cases}
$$

where $d=2$ for two-dimensional space, $\theta=1$ for the multiquadric, $\rho$ depends on the value of $\theta$ and $d$ and $\rho=1$ since $\theta \geq d-1, h$ is the distance between adjacent nodes if interpolation nodes are uniformly distributed, $\sigma$ is a positive number and

$$
\xi=\frac{c \sigma+\sqrt{c^{2} \sigma^{2}+4 \sigma(1+\theta+d)}}{4}
$$


Noting that there is no upscattering of neutrons, substituting Eq. (5) into Eqs. (1)-(2), and then collocating at the interpolation nodes yields the matrix equation

$$
\left[\begin{array}{cccc}
\boldsymbol{K}_{1} & 0 & 0 & 0 \\
-\boldsymbol{S}_{1 \rightarrow 2} & \boldsymbol{K}_{2} & 0 & 0 \\
\vdots & \vdots & \ddots & 0 \\
-\boldsymbol{S}_{1 \rightarrow G} & -\boldsymbol{S}_{2 \rightarrow G} & \cdots & \boldsymbol{K}_{G}
\end{array}\right]\left[\begin{array}{c}
\boldsymbol{a}_{1}^{(n)} \\
\boldsymbol{a}_{2}^{(n)} \\
\vdots \\
\boldsymbol{a}_{G}^{(n)}
\end{array}\right]=\left[\begin{array}{c}
\boldsymbol{S}_{1}^{(n-1)} \\
\boldsymbol{S}_{2}^{(n-1)} \\
\vdots \\
\boldsymbol{S}_{G}^{(n-1)}
\end{array}\right]
$$

where

$$
\begin{aligned}
& \boldsymbol{a}_{g}^{(n)}=\left[\begin{array}{llll}
a_{1, g}^{(n)} & a_{2, g}^{(n)} & \cdots & a_{N_{T}, g}^{(n)}
\end{array}\right]^{T} \\
& \boldsymbol{S}_{g}^{(n-1)}=\left[\begin{array}{lllll}
S_{g}^{(n-1)}\left(x_{1}, y_{1}\right) & S_{g}^{(n-1)}\left(x_{2}, y_{2}\right) & \cdots & S_{g}^{(n-1)}\left(x_{N_{T}}, y_{N_{T}}\right)
\end{array}\right]^{T}
\end{aligned}
$$

and the coefficients of the collocation matrix are

$$
\begin{aligned}
& k_{i j, g}=\left\{\begin{aligned}
-D_{g} \nabla^{2} \psi_{j}\left(x_{i}, y_{i}\right)+\Sigma_{r, g} \psi_{j}\left(x_{i}, y_{i}\right), & 1 \leq i \leq N_{D}+N_{B} \\
\psi_{j}\left(x_{i}, y_{i}\right), & N_{D}+1 \leq i \leq N_{D}+N_{V} \\
\frac{\partial \psi_{j}}{\partial x}\left(x_{i}, y_{i}\right), & N_{D}+N_{V}+1 \leq i \leq N_{D}+N_{V}+N_{R, x} \\
\frac{\partial \psi_{j}}{\partial y}\left(x_{i}, y_{i}\right), & N_{D}+N_{V}+N_{R, x}+1 \leq i \leq N_{T}
\end{aligned}\right. \\
& s_{i j, g^{\prime} \rightarrow g}=\Sigma_{s, g^{\prime} \rightarrow g} \psi_{j}\left(x_{i}, y_{i}\right),
\end{aligned}
$$

where $1 \leq j \leq N_{T}$ and $N_{R, x}$ is the number of reflective nodes for which $\partial \phi_{g} / \partial x=0$. It is seen from the first entry of Eq. (11) that the neutron diffusion equation is collocated on the boundary of the domain. Solution of Eq. (9) yields $a_{j, g}, j=1, \ldots, N_{T}, g=1, \ldots, G$, and hence the numerical result.

\section{Results and Discussion}

Three problems are considered in examining the performance of the RBF collocation method utilizing optimum shape parameters. First case is a one-group external source problem, and the remaining problems are one and two-group fission source cases. All calculations are performed with MATHEMATICA 11 on a $2.5 \mathrm{GHz}$ computer. Uniformly distributed sets of interpolation nodes are employed and arbitrary precision arithmetic is used to take full advantage of the optimum shape parameters. For the calculation of optimum $c, \sigma$ is taken as $\sigma=1$.

For the one-group external source case a trigonometric source is chosen:

$$
s_{\text {ext }}=\cos \left(\frac{\pi x}{2 a}\right) \cos \left(\frac{\pi y}{2 a}\right), 0 \leq x \leq a, 0 \leq y \leq a
$$


The neutron diffusion equation simplifies to the Helmholtz equation when the neutron energy distribution is reduced to a single energy group and the source is an external one. Then the analytical solution of Eqs. (1)-(2) with the source defined in Eq. (13) becomes

$$
\phi(x, y)=\frac{1}{\Sigma_{a}\left[1+2 L^{2}\left(\frac{\pi}{2 a}\right)^{2}\right]} \cos \left(\frac{\pi x}{2 a}\right) \cos \left(\frac{\pi y}{2 a}\right), 0 \leq x \leq a, 0 \leq y \leq a
$$

where the removal cross section is reduced to the absorption cross section, $\Sigma_{a}$ and $L \equiv \sqrt{D / \Sigma_{a}}$ is the diffusion length.

The size of the square domain is $a=0.25 \mathrm{~m}$, and the nuclear parameters are $D=0.0177764 \mathrm{~m}$ and $L=0.111232 \mathrm{~m}$. The accuracy of the RBF collocation method is tested by calculating the maximum error and root mean square (RMS) error of the neutron flux defined as

$$
\begin{aligned}
\epsilon_{\max } & =\max _{1 \leq i \leq N_{D}+N_{B}}\left[\left|\phi\left(x_{i}, y_{i}\right)-\tilde{\phi}\left(x_{i}, y_{i}\right)\right|\right] \\
\epsilon_{R M S} & =\sqrt{\frac{1}{N_{D}+N_{B}} \sum_{i=1}^{N_{D}+N_{B}}\left[\phi\left(x_{i}, y_{i}\right)-\tilde{\phi}\left(x_{i}, y_{i}\right)\right]^{2}}
\end{aligned}
$$

respectively, where $\tilde{\phi}\left(x_{i}, y_{i}\right)$ is the numerical neutron flux distribution. Calculations are made with 300-precision arithmetic.

The variation of the Madych-Nelson function with respect to $c$ when $h=2.5 \mathrm{~cm}$ is presented in Fig. 1 . For this node distance, the $\mathrm{MN}$ function is minimized when $c_{o p t}=2.40367$. The optimum value of the shape parameter tends to increase with a decrease in $h$ [9] and for the external source problem considered $c_{\text {opt }}$ follows this trend up to $a / h=10$. On the other hand, for $a / h \geq 15$ the MN function takes its minimum value at $12 a \rho=3$.

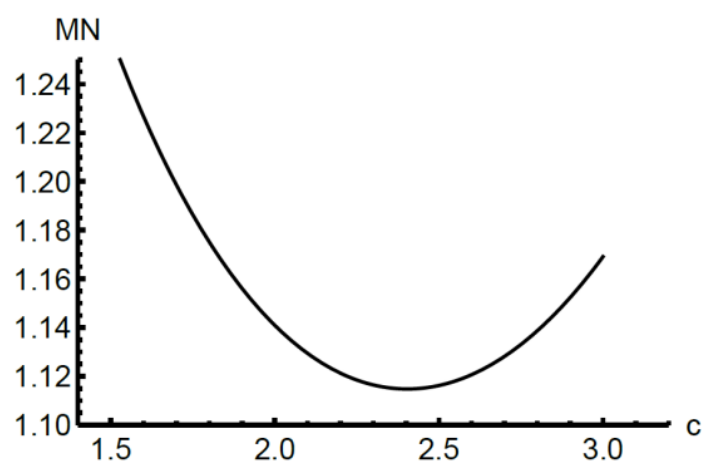

Figure 1. The variation of the Madych-Nelson function with the shape parameter for the external source problem when $\mathrm{h}=2.5 \mathrm{~cm}$

Fig. 2 illustrates the effect of node distance on the maximum and RMS errors of the collocation solution on a semi logarithmic scale. The results show that the method converges exponentially, and it is possible to obtain a very high level of accuracy even with sparse node distributions. The maximum and RMS errors decrease with decreasing $h$, and it is found that $\epsilon_{\max }=5.006 \times 10^{-60}$ and $\epsilon_{R M S}=5.171 \times 10^{-63}$ when $a / h=50$. It should be noted that the levels 
of accuracy presented in Fig. 2 is a consequence of not only the optimum shape parameters but also the use of high precision arithmetic.

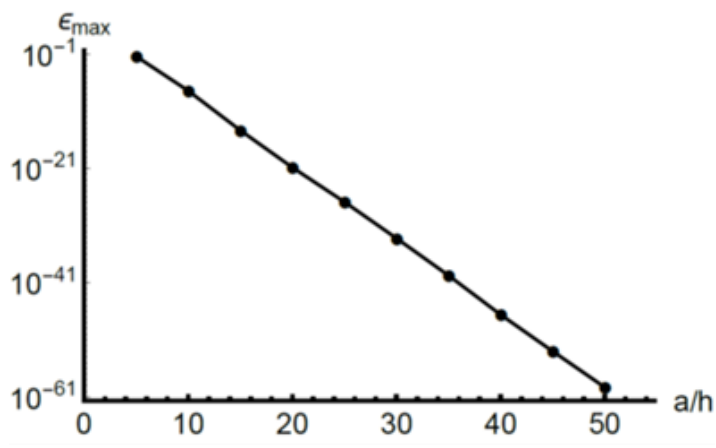

(a)

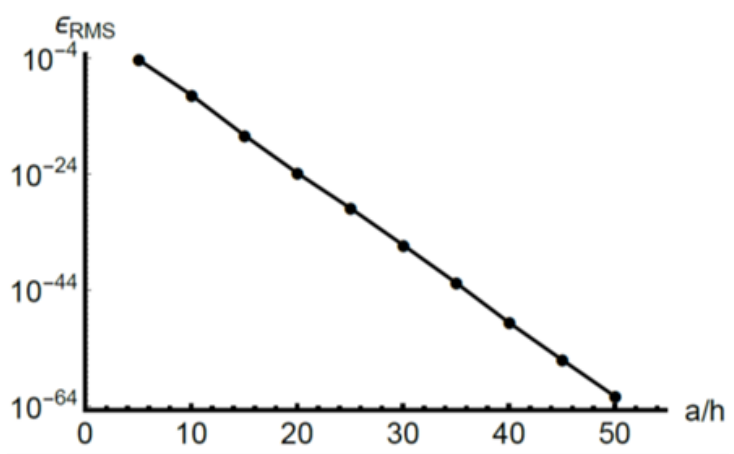

(b)

Figure 2. Convergence curves for the external source problem.

The second problem is a one-group fission source case where $a=0.5 \mathrm{~m}, D=0.0177764 \mathrm{~m}, \Sigma_{a}=1.43676 \mathrm{~m}^{-1}, \Sigma_{f}=$ $1.04869 \mathrm{~m}^{-1}$, and $v=2.5$. These parameters yield an analytical $k$ value of approximately 1.466574 . The criterion of performance for this type of problem is the relative percent error in the multiplication factor defined as

$$
\epsilon_{k}=\frac{|k-\tilde{k}|}{k} \times 100
$$

where $\tilde{k}$ is the numerical multiplication factor. Numerical tests are performed with 500-precision arithmetic, and the convergence criterion for fission source iteration is chosen to be $10^{-30}$ in order to make it possible to be compatible with the high precision computations.

The relative percent error in $k$, CPU time, and optimum shape parameters of the RBF collocation solution for the onegroup fission source problem are given in Table 1. The optimum value of $c$ increases with a decrease in $h$, except at $a / h=5$ for which the MN function takes its minimum value for $24 \rho h=2.4$. Similar to the error behavior of the external source problem, high levels of accuracies are observed. Calculations are limited to $a / h=30$ due to the fact that the high precision arithmetic and the associated fractional convergence criterion cause the CPU time grows in a fast manner as $h$ decreases.

Table 1. $\epsilon_{k}$, CPU time, and optimum shape parameters for the one-group fission source problem.

\begin{tabular}{cccc}
\hline$a / h$ & $\epsilon_{k}$ & $\mathrm{CPU}$ & $c_{\text {opt }}$ \\
\hline 5 & $8.879 \times 10^{-5}$ & 5.68 & 2.4 \\
10 & $4.083 \times 10^{-8}$ & 67.79 & 1.37717 \\
15 & $4.301 \times 10^{-13}$ & 421.163 & 1.83449 \\
20 & $7.085 \times 10^{-20}$ & 1499.653 & 2.40367 \\
25 & $2.196 \times 10^{-27}$ & 4067.787 & 3.04463 \\
30 & $4.216 \times 10^{-29}$ & 9812.435 & 3.72107 \\
\hline
\end{tabular}

The final problem is a two-group fission source case. The size of the domain is $0.6 m$, and the nuclear parameters are given in Table 2 . The analytical value of $k$ for this configuration is approximately 1.152256 . The numerical results are 
obtained with 500-precision arithmetic. The variation of the relative percent error in $k$ with node distance is illustrated in Fig. 3. A high level of accuracy for the two-group case is achieved with the meshless collocation method. However, the results indicate that the convergence is slower when $4 \leq a / h \leq 10$ since the $\mathrm{MN}$ function is minimized at $c_{\text {opt }}=$ $24 \rho h$ for this interval, which means that the value of the shape parameter decreases with increasing $a / h$. For $a / h \geq 12$, the $\mathrm{MN}$ function takes its minimum value in $[24 \rho h, 12 a \rho)$ corresponding to an increasing trend in $c_{\text {opt }}$ with increasing $a / h$.

Table 2. Two-group nuclear data.

\begin{tabular}{ccccccc}
\hline Group & $D(m)$ & $v$ & $\Sigma_{f}\left(m^{-1}\right)$ & $\Sigma_{r}\left(m^{-1}\right)$ & $\Sigma_{s, g \rightarrow g+1}\left(m^{-1}\right)$ & $\chi$ \\
\hline 1 & 0.046245 & 2.65 & 3.3 & 13.552 & 9.776 & 0.575 \\
2 & 0.046245 & 2.55 & 3.376 & 8.228 & - & 0.425 \\
\hline
\end{tabular}

The value of the optimum shape parameter is related to the size of the domain and the numerical tests on one- and twogroup fission source cases reveal that the iterative algorithm is sensitive to this factor. Depending on the value of $a$ the fission source iteration may diverge or result with a wrong multiplication factor. This issue can be dealt by decreasing the value of the shape parameter via increasing the value of $\sigma$.

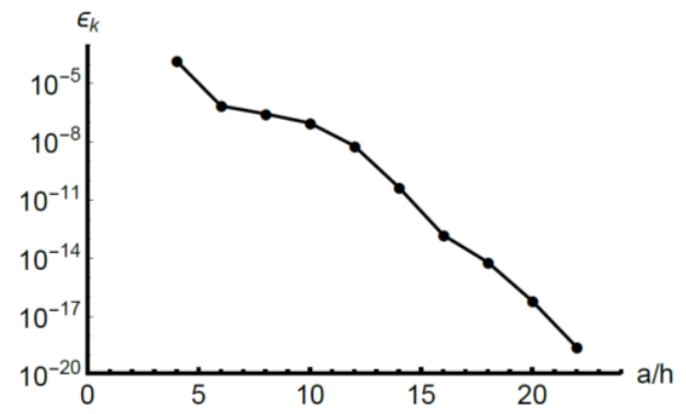

Figure 3. The variation of $\epsilon_{k}$ with $h$ for the two-group fission source problem.

\section{Conclusion}

In this study, the multigroup neutron diffusion equation is solved numerically by the meshless radial basis function collocation method using optimum shape parameters. The multiquadric is chosen as the radial function, and the optimum shape parameters are determined by minimizing the Madych-Nelson function. The optimum value of the shape parameter depends on the node distance, and the Madych-Nelson function may take its minimum value either within or on the endpoints of the optimization interval. The performance of the method is tested by studying on an external and two fission source problems. Calculations are carried out with high precision arithmetic to take advantage of the optimum shape parameters. For all the cases, the meshless collocation method resulted with an exponential convergence rate and a very high level of accuracy. The accuracy of the method originates from the combined effect of using optimum shape parameters together with the employment of high precision arithmetic. Although the central processing unit time of computation increases sharply with decreasing node distance, the results show that the neutron flux distribution and the multiplication factor can be determined accurately even with a few number of interpolation nodes. However, for the fission source cases it is found that the iterative algorithm may diverge or result with incorrect multiplication factors, but this problem can be dealt with by decreasing the value of the optimum shape parameter. 


\section{References}

[1] Kansa, E.J. (1986). Application of Hardy's multiquadric interpolation to hydrodynamics, In: R. Crosbie, P. Luker (Eds.), Proceedings of the 1986 Summer Computer Simulation Conference, San Diego, Society for Computer Simulation, 4:111-117.

[2] Madych, W.R. (1992). Miscellaneous error bounds for multiquadric and related interpolators. Computers and Mathematics with Applications, 24:121-138.

[3] Hardy, R.L. (1971). Multiquadratic equations for topography and other irregular surfaces. Journal of Geophysical Research, 76:1905-1915.

[4] Franke, R. (1982). Scattered data interpolation tests of some methods. Mathematics of Computation, 38:181200.

[5] Fasshauer, G.E. (2002). Newton iteration with multiquadrics for the solution of nonlinear PDEs. Computers and Mathematics with Applications, 43:423-438.

[6] Rippa, S. (1999). An algorithm for selecting a good value for the parameter $\mathrm{c}$ in radial basis function interpolation. Advances in Computational Mathematics, 11:193-210.

[7] Esmaeilbeigi, M. and Hosseini, M.M. (2014). A new approach based on the genetic algorithm for finding a good shape parameter in solving partial differential equations by Kansa's method. Applied Mathematics and Computation, 249:419-428.

[8] Koupaei, J.A., Firouznia, M. and Hosseini, M.M. (2018). Finding a good shape parameter of RBF to solve PDEs based on the particle swarm optimization algorithm. Alexandria Engineering Journal, 57:3641-3652.

[9] Luh, L.T. (2019). The choice of the shape parameter-A friendly approach. Engineering Analysis with Boundary Elements, 98:103-109.

[10] Tanbay, T. and Ozgener, B. (2013). Numerical solution of the multigroup neutron diffusion equation by the meshless RBF collocation method. Mathematical and Computational Applications, 18:399-407.

[11] Tanbay, T. and Ozgener, B. (2014). A comparison of the meshless RBF collocation method with finite element and boundary element methods in neutron diffusion calculations. Engineering Analysis with Boundary Elements, 46:30-40.

[12] Tanbay, T. (2018). On the accuracy and stability of the meshless RBF collocation method for neutron diffusion calculations. Journal of Innovative Science and Engineering, 2:8-18.

[13] Fedoseyev, A.I., Friedman, M.J. and Kansa, E.J. (2002). Improved multiquadric method for elliptic partial differential equations via PDE collocation on the boundary. Computers and Mathematics with Applications, 43:439-455. 